Results: S100A11 protein was up-regulated in the muscle from patients with IIMs compared to MG. In PM/DM patients, S100A11 was accumulated in the cytoplasm of regenerating and necrotizing muscle fibers and on the sarcolemma of most fibers. Only some mononuclear infiltrate cells showed S100A11 positivity. In patients with MG, S100A11 was detected on the sarcolemma only. Moreover, S100A11 was increased in plasma of patients with IIMs compared to HC (3.82 [1.47-72.9] vs. $2.84[1.67-11.18] \mathrm{ng} / \mathrm{ml} ; \mathrm{p}=0.0076)$. When divided into groups, patients with DM and CAM showed significant elevation of plasma S100A11 compared to HC (4.10 [2.22-50.81] and 4.52 [1.53-9.11] vs. 2.84 [1.67-11.18] $\mathrm{ng} / \mathrm{ml} ; \mathrm{p}=0.004$ and $\mathrm{p}=0.02$, respectively). Levels of $\mathrm{S} 100 \mathrm{~A} 11$ did not differ between PM and HC. In all patients, S100A11 correlated with LD ( $r=0.300$, $p=0.001)$, CK $(r=0.217, p=0.025)$ and AST $(r=0.343, p<0.001)$, with MYOACT $(r=0.279, p=0.005)$ and with muscle, pulmonary and skin disease activity $(r=0.199$, $p=0.040 ; r=0.224, p=0.025$ and $r=0.307, p=0.001)$. S100A11 levels were elevated in autoantibody-positive group compared to autoantibody-negative group of patients with IIMs (5.22 [1.45-69.29] vs 3.44 [1.58-12.12], $\mathrm{p}=0.010)$.

Conclusions: S100A11 is up-regulated in the circulation of patients with IIMs and correlates with disease activity. Accumulation of S100A11 in the muscle of patients with myositis may indicate its potential role in the process of cell necrosis and fiber regeneration.

Acknowledgements: Supported by grant 15-34065A of the Agency for Healthcare Research of the Czech Republic and MHCR 023728.

Disclosure of Interest: None declared

DOI: 10.1136/annrheumdis-2017-eular.4758

\section{THU0059 ONCOSTATIN M INDUCES INFLAMMATION AND DIFFERENTIALLY REGULATES TNF ALPHA-INDUCED PRO-INFLAMMATORY MECHANISMS AND NOTCH SIGNALLING IN THE RA JOINT}

M.M. Hanlon ${ }^{1}$, D. Veale ${ }^{2}$, S. Wade ${ }^{1}$, M. Biniecka ${ }^{2}$, U. Fearon ${ }^{1}$, T. McGarry ${ }^{1}$ ${ }^{1}$ Molecular Rheumatology, Trinity Biomedical Sciences Institute, Trinity College Dublin; ${ }^{2}$ Centre for Arthritis and Rheumatic Diseases, St Vincents University Hospital, University College Dublin, Dublin, Ireland

Background: Oncostatin M (OSM) is a pleiotropic cytokine, highly expressed in the RA joint that displays both agonistic and antagonistic effects depending on the inflammatory microenvironment. This study examines the effect of OSM on inflammation, the Notch-1 signalling pathway which plays a critical role in vascular development and angiogenesis and finally on TNF $\alpha$-induced pro-inflammatory mechanisms in Rheumatoid Arthritis.

Objectives: To examine the effect of OSM on cytokine/chemokine production, angiogenesis and the Notch-1 signalling pathway in synovial fibroblasts and endothelial cells and whether OSM potentiates the effects of TNF $\alpha$-induced pro-inflammatory effects.

Methods: Primary RA synovial fibroblasts (RASFC) isolated from RA synovial biopsies obtained at time of knee arthroscopy and human dermal microvascular endothelial cells (HMVEC) were grown to confluence. RASFC and HMVEC were cultured with OSM (10ngml) alone or in combination with increasing concentrations of TNF $\alpha(0.01-1 \mathrm{ng} / \mathrm{ml})$. IL-6, IL-8, RANTES, GRO $\alpha$ and MCP1 cytokines/chemokines were quantified in culture supernatants by ELISA. Functionally, angiogenesis and invasion were assessed by matrigel tube formation and Transwell invasions assays respectively, and VEGF in cell lysates quantified by Real-time PCR. Finally, Notch-1, its ligands Delta-like-ligand 4 (DLL-4) and Jagged-1 (Jag-1) and downstream transcriptional repressors - Hey-1 and Hey-2 were quantified by Real-time PCR.

Results: OSM alone significantly induced IL-6 and MCP-1 while inhibiting IL-8 and GRO $\alpha$ in RASFC and HMVEC culture supernatants, compared to basal control. OSM alone induced RANTES expression in HMVEC with little effect observed for RASFC. OSM potentiated the effect of increasing concentrations of TNF $\alpha$ on IL- 6 and MCP-1 secretion from RASFC and HMVEC. Conversely OSM significantly inhibited TNF $\alpha$-induced IL-8 and GRO $\alpha$ secretion from both RASFC and HMVEC. Interestingly, OSM significantly inhibited TNFa-induced RANTES expression in HMVEC yet conversely potentiated this effect in RASFC. At a functional level, OSM induced both RASFC and HMVEC invasion and induced network formation and VEGF expression in HMVEC. OSM significantly induced Notch-1 in RASFC and HMVEC in a time dependent manner, but interestingly differentially regulated the Notch-1 ligands, with induction of Jag-1 only observed in RASFC, and induction of DLL-4 only observed in HMVEC. Finally OSM differentially regulated Notch-1 downstream transcriptional repressors in HMVEC, significantly inducing Hey-2 while simultaneously inhibiting Hey-1.

Conclusions: OSM is a pleiotropic cytokine that displays divergent effects with both pro- and anti-inflammatory mechanisms within the inflamed joint, effects that appear to be dependent on cell type and the inflammatory microenvironment. Targeting OSM or downstream signalling pathways may lead to new potential therapeutic strategies or adjuvant therapies, particularly for those patients who have sub-optimal responses.

Disclosure of Interest: None declared

DOI: 10.1136/annrheumdis-2017-eular.6334

\section{THU0060 T CELL-DERIVED IL-17A AND IL-17F DRIVE BONE FORMATION FROM HUMAN PERIOSTEAL STEM CELLS: IMPLICATIONS FOR ENTHESOPHYTE FORMATION}

M. Shah ${ }^{1}, 2$, A. Maroof ${ }^{1}$, R. Al-Hosni ${ }^{3}$, P. Gikas ${ }^{4}$, N. Gozzard ${ }^{1}$, S. Shaw ${ }^{1}$ S. Roberts ${ }^{1,2}$. ${ }^{1}$ UCB Pharma, Slough; ${ }^{2}$ University College London; ${ }^{3}$ Institute of Orthopaedics and Musculoskeletal Science; ${ }^{4}$ The Royal Orthopaedic Hospital, London, United Kingdom

Background: Pathological bone formation associated with spondyloarthropathies $(\mathrm{SpA})$ is a major cause of structural tissue damage causing permanent disability. A paucity of in vitro models that faithfully replicate human skeletal biology has impeded research into the cellular and molecular triggers for this osteoimmunological phenomenon. Nevertheless, clinical and animal studies have defined IL-17 signalling as a key regulator of $\mathrm{SpA}$ disease; however, the role of IL-17 in bone pathology is unclear. IL-17-producing $\gamma \delta$-T cells have a critical function in periosteal bone formation for fracture repair ${ }^{1}$. The periosteum has also been implicated in pathological bone formation during SpA disease progression².

Objectives: To investigate IL-17 signalling in the context of pathological bone formation using a biomimetic human periosteum derived stem cell (hPDSC) model of osteogenic differentiation.

Methods: hPDSCs were obtained through enzymatic digestion of periosteal biopsies from patients undergoing orthopaedic surgery. Expanded cultures were then treated with recombinant human IL-17A, IL-17F, or both over 96h. The expression of gene markers was then evaluated. Alternatively, hPDSCs were stimulated using a biomimetic protocol in combination with IL-17A and IL-17F, or human T-cell supernatants (SNs) (as a surrogate disease-like inflammatory milieu). Antibodies with strong-affinity to IL-17A, IL-17F, or bimekizumab (a humanised monoclonal IgG1 antibody with strong affinity for both IL-17A and IL-17F) were used to define the role of these cytokines in the SNs. Expression of osteogenic markers and matrix mineralisation was assessed to define in vitro bone formation.

Results: Under basal conditions IL-17A and IL-17F significantly up-regulated IL-6 expression. Additionally, IL-17A and IL-17F transiently enhanced the expression of the osteogenic transcription factor RUNX-2. When IL-17 cytokines were combined in a biomimetic differentiation protocol, both IL-17A and IL-17F promoted osteogenic differentiation. Importantly, IL-17F enhanced the expression of most osteogenic markers to a greater extent than IL-17A alone following 9 days' exposure. Conversely, IL-17A treatment resulted in elevated in vitro mineralisation vs IL-17F. Th17 and $\gamma \delta-T$ cell SNs potently enhanced hPDSC osteogenic differentiation and mineralisation. Whilst $I L-6$ expression and in vitro bone formation were blocked by neutralisation of IL-17A or IL-17F, dual neutralisation of IL-17A and IL-17F in the inflammatory milieu exhibited the greatest effect on most of the tested parameters. Conclusions: These data show that both IL-17A and IL-17F enhance in vitro osteogenic differentiation and bone formation from hPDSCs. The source of these cytokines has not been established but is likely to involve entheseal resident $\gamma \delta$-T cells. We propose that following their release, IL-17A and IL-17F drive pathological bone formation resulting in enthesophytes at the enthesis/periosteum interface. Current therapeutics display limited efficacy in blocking enthesophyte formation, hence inhibition of both IL-17A and IL-17F offers an attractive therapeutic strategy to prevent this debilitating feature of $\mathrm{SpA}$.

References:

[1] Ono, et al. Nat Commun 2016;7:10928.

[2] Lories, et al. Arthritis Res Ther 2009;11:221.

Disclosure of Interest: M. Shah Grant/research support from: UCB Pharma, Employee of: UCB Pharma, A. Maroof Employee of: UCB Pharma, R. Al-Hosni: None declared, P. Gikas: None declared, N. Gozzard Employee of: UCB Pharma, S. Shaw Employee of: UCB Pharma, S. Roberts Employee of: UCB Pharma DOI: 10.1136/annrheumdis-2017-eular.3826

\section{THU0061 REGULATION OF OSTEOCLAST RECRUITMENT AND ACTIVATION BY RESOLVIN D1}

H.A. Benabdoune, P. Rondon, Q. Shi, J.C. Fernandes, H. Fahmi, M. Benderdour Research Centre, Sacré-Coeur Hospital of Montreal, Room K-3045, Montreal, Canada

Background: Resolvin-D1 (RvD1) is a derivative of omega-3 fatty acids and a potent anti-inflammatory agent synthesized during the resolution phase of inflammation. In human cartilage, we recently reported that RvD1 strongly inhibited a number of factors involved in inflammation, catabolism, oxidative stress, and apoptosis.

Objectives: The overall objective of this study is to further investigate RvD1 effects on bone metabolism in vitro.

Methods: First, murine macrophages RAW267.4 were used to assess osteoclasts (OC) recruitment and bone resorption. RAW264.7 cells were incubated with 50 $\mathrm{ng} / \mathrm{ml}$ LPS with or without RvD1 $(0-10 \mu \mathrm{M})$ for 48 hours. Cell viability was verified with the MTS test. OC phenotype markers, namely TRAP and cathepsin-K, were assessed by western blot, enzymatic staining and immunocytochemistry. Levels of TNF- $\alpha$, IL-1 $1 \beta$, IL-6, IL-10, were measured by ELISA, and PGE 2 levels by EIA. NO release was measured by Greiss reaction.

To investigate bone resorption, RAW264.7 cells were seeded in hydroxyapatite plates, then treated with $50 \mathrm{ng} / \mathrm{ml}$ LPS with or without RvD1 $(0.5$ and $1 \mu \mathrm{M})$ for 48 hours. Plot formation was assessed by Von Kossa staining. 